\title{
A new shape calculus for CutFEM discretizations
}

\author{
Martin Berggren* \\ ${ }^{*}$ Department of Computing Science \\ Umeå University, Sweden \\ e-mail: martin.berggren@cs.umu.se,web page: https://people.cs.umu.se/martinb
}

\begin{abstract}
A basic task in any gradient-based shape optimization project is the sensitivity analysis, that is, the calculation of derivatives of objective functions and constraints with respect to domain variations. The most fundamental issue is to give a precise meaning to "domain variations" and how to differentiate with respect to a geometric property of this kind. The dominating strategy in practice is to utilize transformations $T_{t}$ of the domain, generated by a vector field $\boldsymbol{V}$ (usually referred to as a "velocity"), of the form $T_{t}=I+t \boldsymbol{V}$ for $t \in \mathbb{R}$. There will be one velocity field per design variable. Integrals over the perturbed domain can then be converted, using a change of variables in terms of $T_{t}$, into integrals over the unperturbed domain with the Jacobian of the transformation occurring in the integrand. The transformed integral then becomes a functional with respect to $\boldsymbol{V}$, which may then be differentiated using standard variational calculus.

When carrying out shape sensitivity analysis in the context of a boundary-value problem for a partial differential equation, the final expression for the directional derivative with respect to $\boldsymbol{V}$ of an objective function or constraint comes in two different forms: as a volume integral, involving the full field $\boldsymbol{V}$, or as a boundary integral, involving only the normal trace of $\boldsymbol{V}$ on the boundary. In the discrete case, after finite-element discretization, the volume form of the directional derivative turns out to be the exact derivative of the discrete objective function, provided that the mesh is deformed according to the velocity field used in the formula. The more convenient boundary integral form, however, will unfortunately not be exact when using mesh deformations or remeshings due to lack of regularity of the finite-element solution [1].

The dominating strategy for sensitivity analysis, based on domain transformations $T_{t}=I+t \boldsymbol{V}$, is thus quite closely associated with and appropriate for shape optimization using deforming meshes. However, embedding methods such as CutFEM [3] are very attractive for shape optimization, precisely because they do not use mesh deformation or remeshing; the computational mesh is fixed in a hold-all domain $D$, the boundary to the computational domain $\Omega \subset D$ is allowed to cut freely throughout $D$, and the computations are (in principle) carried out in $D$. Although a velocity field can artificially be created by extending the movement of the boundary into $\Omega$, no domain transformations are actually used to change $\Omega$ when embedding methods are used. In a recent study using CutFEM [2], we noticed the opposite properties of the volume and surface formulations compared to the description above: the surface formulation of the directional derivative was exact for the discrete objective function, whereas the volume formulation was not! This observation suggests that the standard sensitivity analysis approach is not really appropriate for CutFEM and other embedding approaches.
\end{abstract}

An alternative to the use of transformations to define shape derivatives has recently been introduced by Delfour [4]. His approach generates domain variations not through velocity fields but through dilations into Euclidean space of lower-dimensional objects like points, curves, or surfaces. One attractive property of this approach is that it unifies the concepts of topological and shape derivatives. Differentiation with respect to dilation of a point generates a topological derivative (the sensitivity of a functional with respect to inclusion of a small hole). Differentiation with respect to dilation of the boundary generates the shape derivative with respect to a uniform extension of the boundary in the normal direction. Since no domain transformations are involved, this approach seems more appropriate in the context of embedding methods. 
To generalize the Delfour approach to shape calculus for embedding methods such as CutFEM, we consider here a domain $\Omega$ defined using a level set approach. That is, the domain boundary is given as the points $\boldsymbol{x}$ satisfying $\phi(\boldsymbol{x})=0$, where $\phi$ is a finite-element function defined in a hold all $D \supset \Omega$ using standard $P^{1}$ elements. The boundary is deformed by perturbing the level-set function such that the points $\boldsymbol{x}$ of the perturbed boundary satisfy $\phi_{t}(\boldsymbol{x})=0$, where $\phi_{t}=\phi+t w$, and where $w$ is a standard "hat" basis function. By a generalization of the Delfour approach, the basic integral formulas needed for shape sensitivity analysis can then be derived without the use of domain transformations. For instance, for the objective function

$$
J(\phi)=\int_{\Omega} f d V
$$

where $f \in H^{1}(D)$ is given, and $\phi$ is the finite-element level-set function defining $\Omega$, the semidifferential ( $J$ will only be one-sided differentiable in general) with respect to level-set perturbations with basis function $w$ is

$$
d J(\phi ; w)=\lim _{t \rightarrow 0^{+}} \frac{1}{t}\left(J\left(\phi_{t}\right)-J(\phi)\right)=-\int_{\partial \Omega} f \frac{w}{\partial_{n} \phi} d S .
$$

Note that the formula is exact in this discrete case.

Shape sensitivity analysis of boundary integrals can also established using the same mechanism. However, the expression for the semidifferential will be more complicated. The reason is that a boundary defined using a level set of merely $C^{0}$ finite-element functions will contain kinks; the normal field will mesh. The exact semidifferential will thorefore in interfaces.

These formulas for the emidifferential of volume

shape sensitivity analysis for an acoustics problem in the context of a CutFEM discretization. Using

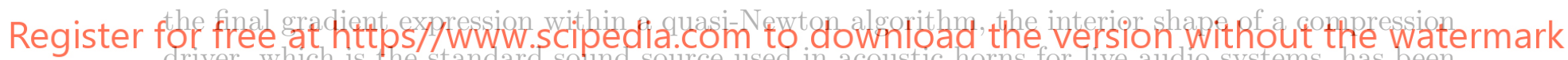
driver, which is the standard sound source used in acoustic horns for live audio systems, has been successfully designed to achieve a favorable frequency response.

\section{REFERENCES}

[1] M. Berggren. A unified discrete-continuous sensitivity analysis method for shape optimization. In W. Fitzgibbon, Y. A. Kuznetsov, P. Neittaanmäki, J. Periaux, and O. Pironneau, editors, Applied and Numerical Partial Differential Equations, volume 15 of Computational Methods in Applied Sciences. Springer, 2010.

[2] A. Bernland, E. Wadbro, and M. Berggren. Acoustic shape optimization using cut finite elements. Internat. J. Numer. Methods Engrg., 113:432-449, 2018.

[3] E. Burman, S. Claus, P. Hansbo, M. G. Larson, and A. Massing. CutFEM: Discretizing geometry and partial differential equations. Internat. J. Numer. Methods Engrg., 104:472-501, 2015 .

[4] M. D. Delfour. Topological derivative: Semidifferential via Minkowski content. J. Convex Anal., 25(3):957-982, 2018. 